\title{
Engrailed-2 promotes a malignant phenotype of esophageal squamous cell carcinoma through upregulating the expression of pro-oncogenic genes
}

\author{
Yong Cao ${ }^{1}$, Xiaoyan Wang ${ }^{1}$, Li Tang ${ }^{1}$, Yan Li ${ }^{1}$, Xueqing Song ${ }^{1}$, Xu Liu ${ }^{1}$, Mingying Li ${ }^{1}$, Feng Chen ${ }^{1}$, Haisu Wan \\ ${ }^{1}$ Experimental Medicine Center, The Affiliated Hospital of Southwest Medical University, Luzhou, Sichuan, China \\ Corresponding Author: Haisu Wan \\ Email address: whssyzx@swmu.edu.cn
}

Background. A number of homeobox genes have been implicated in the development of various cancers. However, the role of engrailed 2 (EN2), a member of the homeobox gene superfamily, in esophageal squamous cell carcinoma (ESCC) remains unknown.

Methods. The expression of EN2 was examined using quantitative real-time PCR and immunohistochemistry. A stable cell line was established to express exogenous EN2 using a lentivirus system. The malignant phenotype was analyzed with proliferation, clonogenicity, wound-healing, and invasion assays. The CRISPR/Cas9 system was adopted to deplete endogenous EN2. RNA profiling was performed using gene expression microarray. The ShRNA-mediated method was used to knock down the expression of SPARC. The structure-function relationship was determined using site-directed mutagenesis.

Results. EN2 is highly expressed in ESCC. The malignant phenotype of the ESCC cell line was amplified by an overexpression of EN2 but was attenuated by a disruption of EN2. RNA profiling analysis revealed that distinct sets of genes were modulated by the expression of EN2 in various ESCC cell lines, and oncogenes were among these. EN2 greatly increased the expression of SPARC in Eca109. Site-directed mutagenesis revealed that the induction of SPARC was closely correlated with the protumor function of EN2. ShRNA-mediated knockdown of SPARC attenuated the malignant phenotype of EN2-infected cells. These data suggest that SPARC is crucial for mediating the protumor function of EN2.

Discussion. EN2 has an oncogenic function in ESCC that is mediated by upregulating the expression of pro-oncogenic genes downstream. EN2 may be a potentially act as a diagnostic marker or therapeutic target for ESCC treatment in the future. 
1 Engrailed-2 promotes a malignant phenotype of esophageal squamous cell carcinoma

2 through upregulating the expression of pro-oncogenic genes

3 Yong Cao ${ }^{1}$, Xiaoyan Wang ${ }^{1}$, Li Tang ${ }^{1}$, Yan $\mathrm{Li}^{1}$, Xueqing Song ${ }^{1}$, Xu Liu ${ }^{1}$, Mingying $\mathrm{Li}^{1}$, Feng

4 Chen $^{1}$ and Haisu Wan ${ }^{1}$

61 Experimental Medicine Center, The Affiliated Hospital of Southwest Medical University,

7 Luzhou, Sichuan, China

9 Corresponding Author:

10 Haisu Wan ${ }^{1}$

11 No. 25, Taiping Street, Luzhou, Sichuan, 646000, China.

12 Email address: whssyzx@swmu.edu.cn

13 Abstract

14 Background. A number of homeobox genes have been implicated in the development of various 15 cancers. However, the role of engrailed 2 (EN2), a member of the homeobox gene superfamily, 16 in esophageal squamous cell carcinoma (ESCC) remains unknown.

17 Methods. The expression of EN2 was examined using quantitative real-time PCR and 18 immunohistochemistry. A stable cell line was established to express exogenous EN2 using a lentivirus system. The malignant phenotype was analyzed with proliferation, clonogenicity, wound-healing, and invasion assays. The CRISPR/Cas9 system was adopted to deplete endogenous EN2. RNA profiling was performed using gene expression microarray. The ShRNAmediated method was used to knock down the expression of SPARC. The structure-function relationship was determined using site-directed mutagenesis.

24 Results. EN2 is highly expressed in ESCC. The malignant phenotype of the ESCC cell line was amplified by an overexpression of EN2 but was attenuated by a disruption of EN2. RNA profiling analysis revealed that distinct sets of genes were modulated by the expression of EN2

27 in various ESCC cell lines, and oncogenes were among these. EN2 greatly increased the 
28 expression of SPARC in Eca109. Site-directed mutagenesis revealed that the induction of

29 SPARC was closely correlated with the protumor function of EN2. ShRNA-mediated

30 knockdown of SPARC attenuated the malignant phenotype of EN2-infected cells. These data

31 suggest that SPARC is crucial for mediating the protumor function of EN2.

32 Discussion. EN2 has an oncogenic function in ESCC that is mediated by upregulating the expression of pro-oncogenic genes downstream. EN2 may potentially act as a diagnostic marker or therapeutic target for ESCC treatment in the future.

\section{Introduction}

Esophageal squamous cell carcinoma (ESCC) is one of the most common malignancies diagnosed in China and worldwide and is associated with high rates of morbidity and mortality (Lin et al. 2013). The overall 5-year survival rate of ESCC patients is in the low range of 15-25\% (Domper Arnal et al., 2015). Significant efforts have been made to determine the molecular events that occur during cancer development with the expectation that a better understanding of the molecular mechanisms will help to identify more diagnostic markers or therapeutic targets and may improve clinical outcomes.

Homeobox genes in humans are part of a superfamily with approximately 300 members. The associated genes have a homeobox region, approximately 180 base pairs in length, which encodes a highly conserved homeodomain (HD) with a length of about 60 amino acids (Madissoon et al., 2016). The homeobox proteins are transcription factors that can activate or repress the genes downstream (Carnesecchi et al., 2018). The homeodomain is responsible for the sequence-specific binding of the homeobox protein to its target DNA. The three-dimensional structure of the homeodomain consists of three alpha-helices connected by two short loops, which form the structural basis for direct contact with DNA (Fraenkel et al., 1998; Pabo \& Sauer, 1992). Homeobox genes are crucial for normal embryonic development and the maintenance of adult cells (Friedrich et al., 2016; Hueber \& Lohmann, 2008). The aberrant expression of homeobox genes has been observed in a variety of cancers (Mansour \& Senga, 2017; Teo et al., 2016; Zhang et al., 2018).

Engrailed 2 is one member of the homeodomain-containing transcription factor. It is engaged in the development of the nervous system at the embryonic stage and in the establishment and maintenance of the spatial integrity of specific regions of developing tissues (McGrath et al., 
58 59 60

2013; Morgan, 2006). Previous studies have demonstrated that the EN2 gene is highly expressed in cancers of the prostate and breast (Bose et al., 2008; Martin et al., 2005). Our study provides evidence for the role of EN2 in the development of ESCC by its activation of downstream oncogenic genes.

\section{Materials \& Methods}

\section{Cell lines and culture}

The ESCC cell lines, Eca109, Kyse150, and TE-1 were obtained from the Type Culture Collection of the Chinese Academy of Sciences (Shanghai, China). The immortalized human esophageal epithelial cell line (HEEC) and 293FT cell lines were obtained from Shanghai Tongpai Biotechnology Co. LTD (Shanghai, China). HEEC and 293FT cells were maintained in Dulbecco's Modified Eagle's Medium (DMEM) (Invitrogen, Carlsbad, CA, USA) and were supplemented with 10\% fetal bovine serum (FBS) (Invitrogen, Carlsbad, CA, USA). The ESCC cell lines were cultured in Roswell Park Memorial Institute 1640 medium containing 10\% fetal bovine serum. All cells were maintained at $37^{\circ} \mathrm{C}$ in a $5 \% \mathrm{CO} 2$-humidified incubator.

\section{Patients and samples}

We collected 32 pairs of ESCC tissues and their matched adjacent tissues from the Department of Thoracic Surgery of the Affiliated Hospital of Southwest Medical University (Lu Zhou, China). All patients underwent potentially curative surgery without preoperative chemotherapy or radiotherapy. This study was approved by the ethics review board at the Affiliated Hospital of Southwest Medical University (K2018002-R). All of the participants involved in our study provided written informed consent.

\section{Total RNA extraction and RT-PCR assay}

Total RNA was extracted from tumor samples or cells using Trizol reagent (Invitrogen, Carlsbad, CA, USA). Four hundred nanograms of total RNA from each sample were reverse-transcribed into cDNA using the PrimeScript ${ }^{\mathrm{TM}}$ RT reagent kit with gDNA Eraser (Takara, Dalian, China). qRT-PCR was performed using the SYBR Premix Ex Taq II Kit (Takara, Dalian, China) according to the manufacturer's instructions. The specific primers used are shown in Table S1. The expression of GAPDH was used as an internal control. The RNA expression levels of genes were evaluated by the $2-\Delta \Delta$ ct method. All specimens were examined in triplicate.

\section{Construction of the lentiviral vectors and establishment of stable cell lines}


88 The plasmids pVAX-EN2 and pCDH-NEO were constructed in the laboratory and used to 89 generate the EN2-expressing vector. Human EN2 cDNA (G136030, youbio, Hunan, China) was

90

91

92

93

94

95

96

97

98

99

100

101

102

103

104

105

106

107

108

109

110

111

112

113

114

115

116

117

118 amplified and cloned into pVAX1 for pVAX-EN2 (VT1048, youbio, Hunan, China) using the HindIII and XhoI sites. The copGFP sequence was replaced with the neomycin gene in the plasmid pCDH-CMV-MCS-EF1-copGFP (JiRan, Shanghai, China) for pCDH-NEO. The EN2 cDNA was then amplified from pVAX-EN2 and subcloned into the XhaI and BamHI sites of pCDH-NEO. The resultant vector was named pCDH-EN2. The ShRNA vector for the human SPARC gene was created by inserting the shRNA-SPARC sequence into the BamHI and EcoRI sites of pLVX-shRNA2 (JiRan, Shanghai, China). A scramble sequence was inserted into the same sites to establish a control vector. All of the constructed vectors were verified by Sanger sequencing.

The related sequences are as follows:

ShRNA-SPARC sense: 5'-gatcc cggcg gttgt tcttt cctca cattt caaga gaatg tgagg aaaga acaac cgttt ttt-3' and antisense: 5'-aatta aaaaa cggtt gttct ttcct cacat tctct tgaat gtgag gaaag aacaa ccgcc gg-3'; scramble sense: 5'-gatcc cggta caaca gccac aacgt ctatt caaga gatag acgtt gtgge tgttg tattt ttt-3' and antisense: 5'-aatta aaaaa tacaa cagcc acaac gtcta tctct tgata gacgt tgtgg ctgtt gtacc gg-3'.

The stable cell lines were established by cotransfecting 293FT cells with the correct respective lentiviral vector, which was either pCDH-EN2 or pCDH-NEO (control); and the pSPAX2 (VT1444, youbio, Hunan, China) and pMD2.G (VT1443, youbio, Hunan, China) package plasmids using PEI reagent (Polysciences, Warrington, PA, USA), following standard laboratory protocol (Toledo et al. 2009). The supernatant containing the virus particles was collected after three days and was used to infect the Eca109 and Kyse150 cell lines. The positive cell clones were selected using G418 (500 $\mu \mathrm{g} / \mathrm{mL}$; Thermo Fisher Scientific, Waltham, MA USA) and were named EN2-Eca109, NEO-Eca109, EN2-Kyse150, and NEO-Kyse150. To assess the function of SPARC, shRNA-SPARC was lentivirally infected into either EN2-Eca109 or NEO-Eca109. The positive cells were selected after 48-hours using flow cytometry based on the expression of EGFP.

\section{Immunofluorescent staining}

Cells were incubated for monolayer growth on glass slides at $37{ }^{\circ} \mathrm{C}, 5 \% \mathrm{CO} 2$ for $24 \mathrm{~h}$. The cell lines were removed from the culture medium and fixed in $4 \%$ paraformaldehyde for 15 minutes at room temperature, followed by an overnight incubation with polyclonal goat anti-EN2

Peer] reviewing PDF | (2019:10:41877:2:0:NEW 23 Jan 2020) 
119 antibody (ab45867; Abcam, Cambridge, UK) diluted (1:100) in 1\% BSA. FITC-conjugated 120 rabbit anti-goat immunoglobulin G (ZSGB-Bio, Beijing, China) was used as the secondary 121 antibody (diluted at 1:200). Cells stained with the secondary antibody were used as the negative 122 control group. Images were collected using a Leica confocal microscope (SP8; Leica, Wetzlar, 123 Germany).

\section{CRISPR construct and clone selection}

125 The DNA sequence surrounding the initial amino acid code in the EN2 locus was selected as the 126 target of this experiment following the guidelines provided on the website of the Feng Zhang 127 Laboratory (http://crispr.mit.edu/). The Cas9 targeting sequence (Cas9-EN2) was formed by 128 annealing two complementary DNA oligos, i.e., sgRNA-EN2-sense and sgRNA-EN2-antisense; 129 this sequence was cloned into the BbsI site of the PX458 vector (\#48138; Addgene, Watertown, 130 MA, USA). The resultant vector, pCas9-EN2, or PX458 vector (control), was transfected into 131 TE-1 cells using Lipofectamine 3000 (Thermo Fisher Scientific, Waltham, MA USA) according 132 to the manufacturer's instructions. EGFP-positive cells were selected after 24 hours by flow 133 cytometry and were cultured in 96-well plates with a concentration of one cell per well.

134 Individual colonies were propagated after a two-week incubation period. The genomic DNA was 135 extracted and PCR-amplified using primer-1 and primer-2. The disruption of the gene was 136 further verified using the mismatch-sensitive T7 endonuclease assay (Bressan et al., 2017; Cong 137 \& Zhang, 2015).

138 The sequences of the oligos and primers used in this experiment were as follows: SgRNA-EN2139 sense: 5'-caccg cggta gcagc ccggg cgaag-3'; sgRNA-EN2-antisense: 5'-aaacc ttcge ccggg ctgct accgc-3'; primer-1: 5'-tgtgc aaga tccga getgt cagag a-3'; and primer-2: 5'-aacgg gtgga caggg tctct 141 acct-3'.

\section{Site-directed mutagenesis}

143 Mutants of the human EN2 gene were generated using our own previously established protocol

144 (Wan et al., 2012). pVAX-EN2 was used as the template. The vector sequence was amplified 145 using a pair of vector primers, VP1 and VP2, which were subjected to Esp 3I digestion. The 146 digested product was self-ligated to generate the expected mutant. The vectors with the expected 147 mutations were verified using Sanger sequencing. The mutated EN2 sequences were then cloned 148 into pCDH-NEO. The vector primers and oligos used in this experiment were as follows: Mut.1- 
149 VP1: 5'-aatta cgtct cagag ccaga acaag cgegc caaga t-3'; Mut.1-VP2: 5'-aatta cgtct cagct cagct 150 cetge gecag gc-3'; Mut.2-VP1: 5'-aattt cgtct caacg ggcaa caaga acacg ctggc-3'; and Mut.2-VP2:

1515 '-aattt cgtct caccg tgaac caaat cttga tctgt gactc gt-3'.

152 Proliferation and colony-formation assay

153 Cell proliferation was examined using both the Cell Counting Kit-8 assay and the live-cell 154 imaging assay. Cells were seeded into 96-well plates at concentrations of 3,000 cells/well using 155 the Cell Counting Kit-8 (CK04, Dojindo, Tokyo, Japan). The culture medium for each cell was 156 replaced at 24,48 , and 72 hours with a solution containing $100 \mu \mathrm{L}$ of fresh medium and $10 \mu \mathrm{L}$ of 157 CCK-8 solution; the cells were incubated at $37^{\circ} \mathrm{C}$ in the dark for one hour. The absorbance (A) 158 value of each well was measured at a wavelength of $450 \mathrm{~nm}$. Cells were seeded in 96-well 159 culture plates for the live-cell image assay at concentrations of 3,000 cells/well and were placed 160 in the IncuCyte Live-Cell Imaging System (Essen Bioscience, Ann Arbor, MI, USA). Growth 161 curves were calculated from confluence measurements using image analysis software. Cells were 162 seeded in six-well plates for the colony formation assay at concentrations of 200 cells/well and 163 the growth medium was changed at three-day intervals. The clones were fixed with 4\% 164 formaldehyde for 10 minutes and stained with freshly prepared diluted Giemsa solution for 15 165 minutes. The clones were then counted and photographed.

\section{Wound healing and matrigel invasion assay}

167 Cells were plated in 96-well plates. After the cells reached 90\%-100\% confluency, a wound was 168 made using the Essen Bioscience 96-pin wound maker (Essen BioScience, Ann Arbor, MI, USA), 169 which generated a cell-free space approximately $600 \mu \mathrm{m}$ wide. The cells were washed twice with 170 PBS to remove the floating cells. $100 \mu \mathrm{L}$ of culture medium containing $1 \%$ FBS was added to 171 the cells for a migration assay. A Matrigel invasion assay was performed in which cells were 172 covered with $50 \mu \mathrm{l}$ of Matrigel solution containing $3 \mathrm{mg} / \mathrm{mL}$ Matrigel (Matrigel Basement 173 Membrane Matrix, 354234, BD Biosciences, San Jose, CA, USA) that was solidified after an 174 incubation period of 30 minutes at $37^{\circ} \mathrm{C}$. The matrix was overlaid with an additional $100 \mu \mathrm{L}$ of 175 normal growth medium. Wound confluency was monitored using the IncuCyte Live-Cell 176 Imaging System. A cell wound healing software module (Essen BioScience, Ann Arbor, MI, 177 USA) (Gujral et al. 2014)was used for image analysis. The wound width was used to calculate 178 the rate of migration and invasion.

\section{Gene expression profiling}


180 Total RNA was extracted using Trizol (Invitrogen, Carlsbad, CA, USA); a total of $500 \mathrm{ng}$ of 181 RNA was used for cDNA synthesis. The following experiment was performed in consultation 182 with Gene Company Ltd. (Hong Kong, China). Gene expression profiles were accessed using the 183 human Affymetrix Clariom S Assay (902926). Microarrays were scanned using the GeneChip ${ }^{\circledR}$ 184 Scanner 3000 7G. The data were analyzed with the robust multichip analysis (RMA) algorithm 185 using default analysis settings and global scaling. Normalization method values were presented 186 according to $\log 2$ RMA signal intensity. R package limma (Ritchie et al., 2015) was used to 187 obtain differential expression genes (DEGs); the cutoff fold-change was set at $>1.5$ or $<-1.5$ 188 and $\mathrm{p}$-value $<0.05$. The DEGs were applied in Gene Ontology analysis by $\mathrm{R}$ package

189 ClusterProfiler (Yu et al., 2012). The transcriptomic data in this article has been uploaded to the 190 GEO repository with the accession number GSE136331.

\section{Western blot assay}

192 The total cellular protein was extracted using a pre-cooled RIPA buffer (Cell Signaling, Danvers, 193 MA, USA) with protease and phosphatase inhibitors. Protein samples were separated by SDS194 PAGE and then transferred to 0.45- $\mu \mathrm{m}$ PVDF membranes (Millipore, Billerica, MA, USA). The 195 membranes were soaked in a blocking solution of $5 \%$ skim milk in Tris-buffered saline (TBS)

196 for 1 hour and incubated overnight at $4^{\circ} \mathrm{C}$ with a primary anti-SPARC antibody (AF941, R\&D), 197 a primary antibody dilution buffer (P0023A, Beyotime) diluted to a ratio of 1:1000, and anti- $\beta$ 198 tubulin antibody (AF1216; R\&D) with a 1:1000 dilution. The blots were subsequently incubated 199 with the homologous HRP-conjugated secondary antibody (Sigma-Aldrich, St. Louis, MO, USA). 200 Immunoreactive bands were detected using ECL reagents (Thermo Fisher Scientific, Waltham, 201 MA, USA).

\section{Immunohistochemistry}

203 Formalin-fixed, paraffin-embedded sections were treated with 3\% H2O2 for 10 minutes after 204 routine deparaffinization in xylene and rehydration in decreasing concentrations of ethanol $205(100 \%, 95 \%, 85 \%$, and $75 \%)$, followed by a period of heating in citrate sodium for antigen 206 retrieval for approximately three minutes. The sections were incubated with 10\% normal goat 207 serum for 20 minutes to block nonspecific reactions after antigen retrieval. The sections were 208 then incubated overnight at $4^{\circ} \mathrm{C}$ with mouse monoclonal antibody, diluted at 1:200, against 209 human EN2 (Santa Cruz Biotechnology, Dallas, TX, USA). After the incubation of the primary 210 antibody, the streptavidin/peroxidase amplification kit (ZSGB-Bio, Beijing, China) was used for 
211 the EN2 antigen-antibody reaction. The sections were treated with diaminobenzidine to promote

212 the appearance of the EN2 signal. Two pathologists were invited to independently score the

213 immunohistochemical signals according to intensity (0-3) and positive percentage (0-100\%).

214 The intensity of positive staining was scored as: 0 , negative $(-)$; 1 , weak $(+) ; 2$, moderate $(++)$;

215 and 3, strong $(+++)$. The expression of EN2 was calculated as the product of the intensity and

216 positive percentage $\times 100$ (IHC score).

217 Statistical analysis

218 SPSS version 16.0 software (IBM Corp., Armonk, NY, USA) was used for statistical analysis.

219 All experimental data are presented as the mean \pm standard deviation from at least three separate

220 experiments. The significance of the mean values between the two groups was analyzed using a

221 two-tailed Student's t-test. Pearson's correlation coefficient was used to analyze the mRNA

222 expression data obtained from qRT-PCR to provide a correlation analysis of EN2 and SPARC

223 expression in the ESCC tissue samples. Statistical p-values of less than 0.05 were considered to

224 be statistically significant.

225 Results

226 EN2 is highly expressed in ESCC tissues

227 Engrailed-2 (EN2), a member of the homeobox superfamily, is highly expressed in a number of 228 cancers, including cancers of the bladder, prostate, and breast (Bose et al., 2008; Martin et al., 229 2005; Morgan et al., 2011; Morgan et al., 2013). The function of EN2 in ESCC is currently 230 unknown and its role was evaluated to further reveal the molecular events linked to the 231 carcinogenesis of ESCC. The expression level of EN2 was first bioinformatically analyzed using 232 two individual datasets from the NCBI GEO database, including GSE130078 and GSE45670 as 233 well as on TCGA database. The mRNA expression of EN2 was found to be significantly 234 elevated in ESCC tissues (Figs. 1A -1C). Surgical specimens derived from ESCC patients were 235 used to examine the expression level of EN2 using quantitative real-time polymerase chain reaction (qRT-PCR) to support these results. The mRNA expression levels of EN2 were

237 significantly upregulated in ESCC tissues compared with the paired adjacent normal tissues ( $\mathrm{p}=$ 238 0.0005; Fig. 1D). Immunohistochemistry was used to analyze the expression of the EN2 protein 239 in tissues. The EN2 protein expression in ESCC was significantly elevated compared with the 240 adjacent normal tissues (Figs. 1E and 1F). These results confirmed that EN2 is highly expressed 241 in ESCC at both the mRNA and protein-specific levels. 


\section{EN2 promotes proliferation and clonogenic abilities of ESCC cell lines}

243 Homeobox genes have emerged as key players in the development of cancer so understanding 244 the function of EN2 in ESCC is of great interest. The mRNA expression levels of EN2 were 245 examined in three ESCC cell lines, namely Eca109, Kyse150, and TE-1, as well as the SHEE 246 cell line, which is an immortalized epithelial cell line derived from the fetal esophageal 247 epithelium induced by HPV 18 E6E7 AAV (Shen et al., 2003). A normal RNA sample was 248 included as a reference, which had an equal molar ratio of total RNA samples from the 32 249 normal samples to that of the tumor samples from the 32 ESCC patients. The mRNA expression 250 of EN2 in Eca109 and Kyse150 was lower than TE-1 (Fig. 2A), and the latter two cell lines were 251 used to study the effect of exogenously expressed EN2 on ESCC cell lines. The lentiviral system 252 was adopted to introduce EN2 into the cell lines. The expression of EN2 transcripts was greatly 253 increased, as was expected (Fig. 2B). The expression of the EN2 protein was confirmed using an 254 immunofluorescence assay (Figs. 2C and 2D). The growth capacity of the EN2-infected cells 255 was evaluated and the growth rates of both EN2-Eca109 and EN2-Kyse150 were significantly 256 increased compared with those of NEO-Eca109 and NEO-Kyse150 (Fig. 2E). Cell growth was 257 also measured using the IncuCyte Zoom live-cell imaging assay, which showed that EN2 258 promoted the proliferation of the two cell lines (Fig. 2F). The effect of EN2 on the colony259 forming ability of Eca109 and Kyse150 was also examined and the results revealed that both 260 EN2-infected cell lines obtained the augmented capacity of colony formation, however, the 261 effect was more apparent in Eca109 (Figs. 2G-2J).

\section{EN2 facilitates migration and invasion of ESCC cell lines}

263 Cancer cells are characterized by their ability to migrate and invade other cells. We tried to 264 determine the effects of EN2 on these malignancy-associated phenotypes. The wound-healing 265 assay was used to examine the effects of EN2 on the migration of ESCC cell lines; EN2266 Kyse150 (Figs. 3A and 3B) and EN2-Eca109 (Figs. 3C and 3D) cells migrated faster than did 267 their respective empty controls. Their invasive ability was examined in the Matrigel using a live268 cell imaging assay. The results revealed that both Kyse150 (Figs. 3E and 3F) and Eca109 (Figs. $2693 \mathrm{G}$ and $3 \mathrm{H}$ ) were more aggressive in the Matrigel after the introduction of exogenous EN2.

270 These results indicate that EN2 could promote the malignant phenotypes of ESCC cells. 


\section{Disruption of EN2 inhibits the proliferation, clonogenicity, migration and invasion of}

\section{ESCC cells}

273 The CRISPR/Cas9 system was used to investigate the biological function of EN2 in ESCC by 274 generating EN2 null cells in TE-1, in which EN2 is highly expressed. The CRISPR/Cas9 275 machinery was designed to destroy the gene structure of the first exon (Fig. 4A). One positive 276 clone was chosen for experimentation. The successful disruption of the EN2 gene was confirmed 277 using the mismatch-sensitive T7 endonuclease assay (Fig. 4B), in which the appearance of bands 278 of $744 \mathrm{bp}$ and $354 \mathrm{bp}$ indicated the expected modification of the target site in the EN2 locus. The 279 PX458 empty vector was transfected into TE-1 cells to generate a control cell line. The 280 expression of the EN2 protein was eliminated in the EN2-disrupted cells as determined by the 281 immunofluorescent staining assay (Fig. 4C). We subsequently assessed whether the disruption of 282 EN2 affects the malignant phenotype of TE-1 cells. TE-1 cells with the disrupted EN2 locus 283 exhibited a reduced growth rate (Fig. 4D). Their colony-forming ability was also weakened when 284 compared with the control (Figs. 4E and 4F). The disruption of EN2 hindered the migration (Figs. $2854 \mathrm{G}$ and $4 \mathrm{H}$ ) and invasive (Figs. 4I and 4J) behavior of TE-1 cells. These results demonstrate that 286 the disruption of EN2 negatively affects the malignant phenotype of TE-1 cells and further 287 supports its oncogenic role in ESCC.

288

289

290

291

292

293

294

295

296

297

298

299

300

\section{EN2 upregulates the expression of SPARC}

EN2 is similar to other homeodomain proteins in its ability to bind to the sequence-specific DNA sites in the chromosome and consequently regulate the downstream genes like other transcription factors. The genetic expression profiling in EN2-Eca109 and EN2-Kyse150 cells was analyzed to determine the molecular mechanisms responsible for their protumor functions. The transcriptomic data were obtained using the Affymetrix clariom $\mathrm{S}$ array to examine their genetic expression. A total of 62 genes were found to be upregulated (Table S2) and 42 genes were downregulated (Table S3) in the EN2-Eca109 cells as compared with the NEO-Eca109 cells (Fig. 5A). A total of 131 genes were found to be upregulated (Table S4) and 249 genes downregulated (Table S5) in the EN2-Kyse150 cells compared with the NEO-Kyse150 cells (Fig. S1A)

Gene ontology analysis was conducted to determine the biological function of EN2-regulated genes. The genes upregulated by EN2 in Eca109 are categorized into groups including cAMPdependent kinase inhibition, extracellular matrix binding, protein kinase A activity, growth 
301 receptor binding, cytokine receptor activity, and phosphoric diester hydrolase activity (Fig. 5B).

302 The genes upregulated by EN2 in Kyse150 are categorized into groups including ErbB-2 class

303 receptor binding, extracellular matrix constituent, proteinaceous extracellular matrix, and

304 response to type I interferon (Fig. S1B). The results of the GO analysis of the downregulated

305 genes are shown in Figs. 5C and S1C respectively. These data suggest that EN2 is able to 306 upregulate genes connected to the malignant characteristics of ESCC cells. A segment of gene

307 expression was verified using qRT-PCR analysis (Figs. 5D and S1D). There are many well-

308 documented pro-oncogenic genes (Kojima et al., 2019; Tian et al., 2016; Wu et al., 2019) that are 309 upregulated upon the expression of EN2, implying that EN2 could modulate the expression of

310 the downstream targets genes in a cellular, context-dependent manner.

311 The upregulated oncogenic gene was investigated for its role in mediating the biological function

312 of EN2 using SPARC in the Eca109 cell line. The secreted protein acidic and rich in cysteine

313 (SPARC) is a multi-faceted protein that has a well-documented function in the malignant

314 features of various types of cancer (Chen et al., 2012; Hung et al., 2017). The mRNA level of the 315 SPARC gene increased by about 25 fold with EN2 in Eca109 cells (Fig. 5D). The protein level 316 also increased by approximately 7.5 fold, as determined by western blot testing (Fig. 5E). Our 317 results indicate a drastic induction of SPARC in EN2-Eca109, implying a possible positive correlation between the expression of EN2 and SPARC. We examined both EN2 and SPARC

319 expression in the clinical ESCC samples using qRT-PCR; the results verified the positive 320 correlation of ESCC (Fig. 5F). The expression of SPARC modulated by EN2 was investigated in two other cell lines. The mRNA expression level of SPARC was found to decrease by about $60 \%$ in EN2-null TE-1 cells as determined by qRT-PCR. However, EN2 did not change the expression of SPARC in EN2-Kyse150 cells when compared with its expression in NEOKyse150 cells (Fig. S1E). These data demonstrate that EN2 is able to boost the expression of the SPARC genes in Eca109 and TE-1 but not in Kyse150. The sequence and structure of the homeodomain are highly conserved in the members of the homeobox superfamily. The homeodomain of EN2 has a three-helix structure that directly participates in DNA binding. Two mutants were created to evaluate the significance of the homeodomain in the protumor function of EN2. The mutants each had a small deletion in the homeodomain; the deletions were as follows: mut 1 with a deletion of the residues from 283 to 
332292 and mut 2 with a deletion of the residues from 293 to 302 (Fig. 6A). The sequencing maps

333 around the mutated sites are shown in Fig. 6B. The expression vectors, each containing a

334 mutated EN2, were introduced respectively into Eca109 using the lentivirus system and

335 generating the Mut.1-Eca109 and Mut.2-Eca109 cell lines. The expression of the two mutant

336 transcripts of EN2 was verified using qRT-PCR (Fig. 6C). The effects of the two mutants were

337 evaluated for their ability to proliferate, migrate, and invade the cell line. Both mutants were

338 shown to hinder the promoting effects of EN2 on the proliferation of Eca109 (Fig. 6D). The

339 mutants largely eliminated the enhancing effects of EN2 on the cell's migratory abilities (Figs.

$3406 \mathrm{E}$ and $6 \mathrm{~F}$ ) and invasion in matrigel (Figs. 6G and 6H). The expression status of SPARC in the

341 Mut1-Eca109 and Mut2-Eca109 cell lines was investigated and the results revealed that the

342 mutations in the homeodomain also abrogate the function of EN2 in inducing the expression of

343 SPARC (Figs. 6I and 6J). Our results indicate that the homeodomain is essential for the protumor 344 effect of EN2.

\section{ShRNA-mediated knockdown of SPARC hampers the pro-oncogenic effect of EN2}

346 SPARC may be necessary for mediating the biological functions of EN2. We generated a shRNA

347 lentiviral vector specifically directed against the SPARC transcript to determine whether the

348 EN2-mediated tumor proliferation, migration, and invasion are dependent on SPARC expression.

349 A scramble sequence was used as the control. The introduction of the shRNA vector into NEO-

350 Eca109 and EN2-Eca109 successfully reduced the expression of SPARC proteins by 80\% (Figs.

351 7A and 7B). Knockdown of SPARC significantly reduced the proliferation capacity of EN2-

352 Eca109 (Fig. 7C). We also observed the decreased capacities of the migration and invasion of the

353 shRNA-transfected cells (Figs. 7D-7G). This result demonstrates that SPARC plays a critical

354 role in mediating the biological function of EN2 in the Eca109 cell line.

\section{Discussion}

356 The poor prognosis and high mortality rates of esophageal cancer highlight the need to better

357 understand the molecular events leading to the progression of this disease. We demonstrated that

358 engrailed-2, a member of the homeobox superfamily, may be a potential biomarker in the

359 identification of the malignancy of ESCC and confirmed its oncogenic role in this cancer.

360 Certain members of the homeobox superfamily have been implicated in normal development as

361 well as in the pathological development of cancer. OCT-4 is a homeobox gene well known for its

362 function in generating induced pluripotent stem cells (Bhartiya, 2013; Seiler et al., 2011); this 
363 gene has also been identified as an oncogene known for promoting the features of cancer stem 364 cells (Li et al., 2017; Phiboonchaiyanan \& Chanvorachote, 2017). In addition to OCT-4, an 365 increasing body of evidence reveals that homeobox genes may participate in the promotion of 366 various malignant phenotypes such as the proliferation, migration, and invasion of cancers 367 (Hirao et al., 2019; Miwa \& Kanda, 2019; Rodini et al., 2012; Song et al., 2019). We have 368 previously reported that HoxC6, another member of homeobox superfamily, functions as an 369 oncogene in ESCC (Tang et al. 2019). We therefore performed the correlation analysis using the data retrieved from TCGA, and the result indicates that these two genes function independently (Fig. S2). Nevertheless, these results suggest that HoxC6 and EN2 may complement each other when using as the potential biomarkers in the diagnosis or treatment of ESCC. Additional homeobox genes will likely be recognized as critical factors in the malignancy of ESCC, which may provide clinical opportunities for the diagnosis and treatments of this cancer in the future. EN2 functions as a transcription factor, much like other homeobox genes, binding directly to specific DNA sites in the chromatin via the conserved homeodomain and controlling the expression of downstream targets. In our experiment, EN2 was able to modulate the expressions of hundreds of downstream targets. Although the expression of distinct sets of genes are affected in various ESCC cell lines, it is likely that many of these genes are connected to the mailgnant phenotype of cancer cells. For instance, the expression of SPARC is significantly upregulated upon the expression of EN2 in two of three ESCC cell lines. We further demonstrated that SPARC is capable of mediating the oncogenic effect of EN2 in the Eca109 cell lines. A similar effect was not observed for EN2 on the expression of SPARC in Kyse150 cell lines; however, there were many other oncogenic genes regulated upon the expression of EN2 in this cell line. This phenomenon suggests that the mechanism underlying the biological function of EN2 may be cell context-dependent. Our work confirms the importance of the homeodomain on the promalignant effect of EN2 in ESCC cells. However, the homeodomain alone is not sufficient to determine the DNA-binding specificity of a homeobox gene-encoded protein and the involvement of the amino acid sequence outside of the homeodomain region is also required (van Dijk \& Murre, 1994; Peltenburg \& Murre, 1997). The availability of other co-factors and the accessibility of chromatin also influences the complexity of this system (Porcelli et al. 2019), and 393 may influence the outcome of EN2 expression in various cell lines. Further insight into the molecular mechanisms will help to explain the biological effects of EN2 in ESCC. 
394 The data presented in this work demonstrates that the biological effect of EN2 is correlated with

395 its role in transcription activation. Future studies should seek to clarify the structural basis and 396 components of EN2-containing transcription activation complexes.

\section{Conclusion}

398 We demonstrated that EN2 plays an oncogenic role in ESCC. Further, the biological function of 399 EN2 is mediated via the upregulation of downstream oncogenes. Our work provides more 400 information on the molecular mechanisms underlying the malignancy of this type of cancer. EN2 401 may serve as a potential candidate as a diagnostic marker or therapeutic target of ESCC in the 402 future.

403

404

405

406

407

408

409

410

411

412

413

414

415

416

417

418

419

420

421

422

423

424

425

426

427

428

429

430

\section{Acknowledgements}

We would like to thank our colleagues for their contribution to this study.

\section{References}

Bhartiya D. 2013. Are Mesenchymal Cells Indeed Pluripotent Stem Cells or Just Stromal Cells? OCT-4 and VSELs Biology Has Led to Better Understanding. Stem Cells Int 2013:547501. 10.1155/2013/547501

Bose SK, Bullard RS, and Donald CD. 2008. Oncogenic role of engrailed-2 (en-2) in prostate cancer cell growth and survival. Transl Oncogenomics 3:37-43.

Bressan RB, Dewari PS, Kalantzaki M, Gangoso E, Matjusaitis M, Garcia-Diaz C, Blin C, Grant V, Bulstrode H, Gogolok S, Skarnes WC, and Pollard SM. 2017. Efficient CRISPR/Cas9-assisted gene targeting enables rapid and precise genetic manipulation of mammalian neural stem cells. Development 144:635-648. 10.1242/dev.140855

Carnesecchi J, Pinto PB, and Lohmann I. 2018. Hox transcription factors: an overview of multi-step regulators of gene expression. Int J Dev Biol 62:723-732. 10.1387/ijdb.180294il

Chen J, Wang M, Xi B, Xue J, He D, Zhang J, and Zhao Y. 2012. SPARC is a key regulator of proliferation, apoptosis and invasion in human ovarian cancer. PLoS One 7:e42413. 10.1371/journal.pone. 0042413

Cong L, and Zhang F. 2015. Genome engineering using CRISPR-Cas9 system. Methods Mol Biol 1239:197-217. 10.1007/978-1-4939-1862-1_10

Domper Arnal MJ, Ferrandez Arenas A, and Lanas Arbeloa A. 2015. Esophageal cancer: Risk factors, screening and endoscopic treatment in Western and Eastern countries. World J Gastroenterol 21:7933-7943. 10.3748/wjg.v21.i26.7933

Fraenkel E, Rould MA, Chambers KA, and Pabo CO. 1998. Engrailed homeodomain-DNA complex at 2.2 A resolution: a detailed view of the interface and comparison with other engrailed structures. J Mol Biol 284:351-361. 10.1006/jmbi.1998.2147

Friedrich J, Sorge S, Bujupi F, Eichenlaub MP, Schulz NG, Wittbrodt J, and Lohmann I. 2016. Hox Function Is Required for the Development and Maintenance of the Drosophila Feeding Motor Unit. Cell Rep 14:850-860. 10.1016/j.celrep.2015.12.077 
431

432

433

434

435

436

437

438

439

440

441

442

443

444

445

446

447

448

449

450

451

452

453

454

455

456

457

458

459

460

461

462

463

464

465

466

467

468

469

470

471

472

473

474

475

476

477

478

Gujral TS, Chan M, Peshkin L, Sorger PK, Kirschner MW, and MacBeath G. 2014. A noncanonical Frizzled2 pathway regulates epithelial-mesenchymal transition and metastasis. Cell 159:844-856. 10.1016/j.cell.2014.10.032

Hirao N, Iwata T, Tanaka K, Nishio H, Nakamura M, Morisada T, Morii K, Maruyama N, Katoh Y, Yaguchi T, Ohta S, Kukimoto I, Aoki D, and Kawakami Y. 2019. Transcription factor homeobox D9 is involved in the malignant phenotype of cervical cancer through direct binding to the human papillomavirus oncogene promoter. Gynecol Oncol 155:340-348. 10.1016/j.ygyno.2019.08.026

Hueber SD, and Lohmann I. 2008. Shaping segments: Hox gene function in the genomic age. Bioessays 30:965-979. 10.1002/bies.20823

Hung JY, Yen MC, Jian SF, Wu CY, Chang WA, Liu KT, Hsu YL, Chong IW, and Kuo PL. 2017. Secreted protein acidic and rich in cysteine (SPARC) induces cell migration and epithelial mesenchymal transition through WNK1/snail in non-small cell lung cancer. Oncotarget 8:6369163702. 10.18632/oncotarget.19475

Kojima Y, Kondo Y, Fujishita T, Mishiro-Sato E, Kajino-Sakamoto R, Taketo MM, and Aoki M. 2019. Stromal iodothyronine deiodinase 2 (DIO2) promotes the growth of intestinal tumors in Apc(Delta716) mutant mice. Cancer Sci. 10.1111/cas. 14100

Li L, Wei X, Wu B, Xiao Y, Yin M, and Yang Q. 2017. siRNA-mediated knockdown of ID1 disrupts Nanog- and Oct-4-mediated cancer stem cell-likeness and resistance to chemotherapy in gastric cancer cells. Oncol Lett 13:3014-3024. 10.3892/ol.2017.5828

Lin Y, Totsuka Y, He Y, Kikuchi S, Qiao Y, Ueda J, Wei W, Inoue M, and Tanaka H. 2013. Epidemiology of esophageal cancer in Japan and China. J Epidemiol 23:233-242.

Madissoon E, Jouhilahti EM, Vesterlund L, Tohonen V, Krjutskov K, Petropoulos S, Einarsdottir E, Linnarsson S, Lanner F, Mansson R, Hovatta O, Burglin TR, Katayama S, and Kere J. 2016. Characterization and target genes of nine human PRD-like homeobox domain genes expressed exclusively in early embryos. Sci Rep 6:28995. 10.1038/srep28995

Mansour MA, and Senga T. 2017. HOXD8 exerts a tumor-suppressing role in colorectal cancer as an apoptotic inducer. Int J Biochem Cell Biol 88:1-13. 10.1016/j.biocel.2017.04.011

Martin NL, Saba-El-Leil MK, Sadekova S, Meloche S, and Sauvageau G. 2005. EN2 is a candidate oncogene in human breast cancer. Oncogene 24:6890-6901. 10.1038/sj.onc.1208840

McGrath SE, Michael A, Pandha H, and Morgan R. 2013. Engrailed homeobox transcription factors as potential markers and targets in cancer. FEBS Lett 587:549-554. 10.1016/j.febslet.2013.01.054

Miwa T, and Kanda M. 2019. ASO Author Reflections: Homeobox C10 Influences on the Malignant Phenotype of Gastric Cancer Cell Lines and its Elevated Expression Positively Correlates with Recurrence and Poor Survival. Ann Surg Oncol. 10.1245/s10434-01907405-9

Morgan R. 2006. Engrailed: complexity and economy of a multi-functional transcription factor. FEBS Lett 580:2531-2533. 10.1016/j.febslet.2006.04.053

Morgan R, Boxall A, Bhatt A, Bailey M, Hindley R, Langley S, Whitaker HC, Neal DE, Ismail M, Whitaker H, Annels N, Michael A, and Pandha H. 2011. Engrailed-2 (EN2): a tumor specific urinary biomarker for the early diagnosis of prostate cancer. Clin Cancer Res 17:1090-1098. 10.1158/1078-0432.CCR-10-2410

Morgan R, Bryan RT, Javed S, Launchbury F, Zeegers MP, Cheng KK, James ND, Wallace DM, Hurst CD, Ward DG, Knowles MA, and Pandha H. 2013. Expression of Engrailed-2 (EN2) protein in bladder cancer and its potential utility as a urinary diagnostic biomarker. Eur J Cancer 49:22142222. 10.1016/j.ejca.2013.01.019

Pabo CO, and Sauer RT. 1992. Transcription factors: structural families and principles of DNA recognition. Annu Rev Biochem 61:1053-1095. 10.1146/annurev.bi.61.070192.005201

Peer) reviewing PDF | (2019:10:41877:2:0:NEW 23 Jan 2020) 
479

480

481

482

483

484

485

486

487

488

489

490

491

492

493

494

495

496

497

498

499

500

501

502

503

504

505

506

507

508

509

510

511

512

513

514

515

516

517

518

519

520

521

522

523
Peltenburg LT, and Murre C. 1997. Specific residues in the Pbx homeodomain differentially modulate the DNA-binding activity of Hox and Engrailed proteins. Development 124:1089-1098.

Phiboonchaiyanan PP, and Chanvorachote P. 2017. Suppression of a cancer stem-like phenotype mediated by alpha-lipoic acid in human lung cancer cells through down-regulation of beta-catenin and Oct-4. Cell Oncol (Dordr) 40:497-510. 10.1007/s13402-017-0339-3

Porcelli D, Fischer B, Russell S, and White R. 2019. Chromatin accessibility plays a key role in selective targeting of Hox proteins. Genome Biol 20:115. 10.1186/s13059-019-1721-4

Ritchie ME, Phipson B, Wu D, Hu Y, Law CW, Shi W, and Smyth GK. 2015. limma powers differential expression analyses for RNA-sequencing and microarray studies. Nucleic Acids Res 43:e47. 10.1093/nar/gkv007

Rodini CO, Xavier FC, Paiva KB, De Souza Setubal Destro MF, Moyses RA, Michaluarte P, Carvalho MB, Fukuyama EE, Head, Neck Genome Project G, Tajara EH, Okamoto OK, and Nunes FD. 2012. Homeobox gene expression profile indicates HOXA5 as a candidate prognostic marker in oral squamous cell carcinoma. Int J Oncol 40:1180-1188. 10.3892/ijo.2011.1321

Seiler K, Soroush Noghabi M, Karjalainen K, Hummel M, Melchers F, and Tsuneto M. 2011. Induced pluripotent stem cells expressing elevated levels of sox-2, oct-4, and klf-4 are severely reduced in their differentiation from mesodermal to hematopoietic progenitor cells. Stem Cells Dev 20:1131-1142. 10.1089/scd.2010.0391

Shen ZY, Xu LY, Chen MH, Li EM, Li JT, Wu XY, and Zeng Y. 2003. Upregulated expression of Ezrin and invasive phenotype in malignantly transformed esophageal epithelial cells. World J Gastroenterol 9:1182-1186. 10.3748/wjg.v9.i6.1182

Song C, Han Y, Luo H, Qin Z, Chen Z, Liu Y, Lu S, Sun H, and Zhou C. 2019. HOXA10 induces BCL2 expression, inhibits apoptosis, and promotes cell proliferation in gastric cancer. Cancer Med 8:5651-5661. 10.1002/cam4.2440

Tang L, Cao Y, Song X, Wang X, Li Y, Yu M, Li M, Liu X, Huang F, Chen F, and Wan H. 2019. HOXC6 promotes migration, invasion and proliferation of esophageal squamous cell carcinoma cells via modulating expression of genes involved in malignant phenotypes. PeerJ 7:e6607. 10.7717/peerj.6607

Teo WW, Merino VF, Cho S, Korangath P, Liang X, Wu RC, Neumann NM, Ewald AJ, and Sukumar S. 2016. HOXA5 determines cell fate transition and impedes tumor initiation and progression in breast cancer through regulation of E-cadherin and CD24. Oncogene 35:5539-5551. 10.1038/onc.2016.95

Tian H, Li X, Jiang W, Lv C, Sun W, Huang C, and Chen R. 2016. High expression of AKR1C1 is associated with proliferation and migration of small-cell lung cancer cells. Lung Cancer (Auckl) 7:53-61. 10.2147/LCTT.S90694

Toledo JR, Prieto Y, Oramas N, and Sanchez O. 2009. Polyethylenimine-based transfection method as a simple and effective way to produce recombinant lentiviral vectors. Appl Biochem Biotechnol 157:538-544. 10.1007/s12010-008-8381-2

van Dijk MA, and Murre C. 1994. extradenticle raises the DNA binding specificity of homeotic selector gene products. Cell 78:617-624.

Wan H, Li Y, Fan Y, Meng F, Chen C, and Zhou Q. 2012. A site-directed mutagenesis method particularly useful for creating otherwise difficult-to-make mutants and alanine scanning. Anal Biochem 420:163-170. 10.1016/j.ab.2011.09.019

Peer] reviewing PDF | (2019:10:41877:2:0:NEW 23 Jan 2020) 
524 Wu JI, Lin YP, Tseng CW, Chen HJ, and Wang LH. 2019. Crabp2 Promotes Metastasis of Lung 525

526

527

528

529 Cancer Cells via HuR and Integrin beta1/FAK/ERK Signaling. Sci Rep 9:845. 10.1038/s41598-018-37443-4

Yu G, Wang LG, Han Y, and He QY. 2012. clusterProfiler: an R package for comparing biological themes among gene clusters. OMICS 16:284-287. 10.1089/omi.2011.0118 Zhang B, Li N, and Zhang H. 2018. Knockdown of Homeobox B5 (HOXB5) Inhibits Cell Proliferation, Migration, and Invasion in Non-Small Cell Lung Cancer Cells Through Inactivation of the Wnt/beta-Catenin Pathway. Oncol Res 26:37-44. 10.3727/096504017X14900530835262

532 


\section{Figure 1}

Expression of EN2 is significantly elevated in ESCC.

(A, B) Microarray data retrieved from gene expression omnibus (GEO) repository were utilized to analyze EN2 expression at mRNA level in ESCC tissues including GSE130078 (normal=23, cancer=23) and GSE45670 (normal=10, cancer=28). (C) Data in TCGA database showed higher mRNA level of EN2 in ESCC tissues compared to adjacent normal tissues. (D) Expression of EN2 mRNA in 32 pairs of tumor samples and adjacent normal tissues as determined using qRT-PCR. (E) Representative images of IHC staining of EN2 in ESCC and adjacent normal tissues. (F) Expression of EN2 protein as determined by $\mathrm{IHC} \square \mathrm{n}=24 \square$. The $\mathrm{IHC}$ score of EN2 was calculated as the staining intensity $(0,1,2$, or 3$) \times$ positive percentage $\times$ 100. 
A

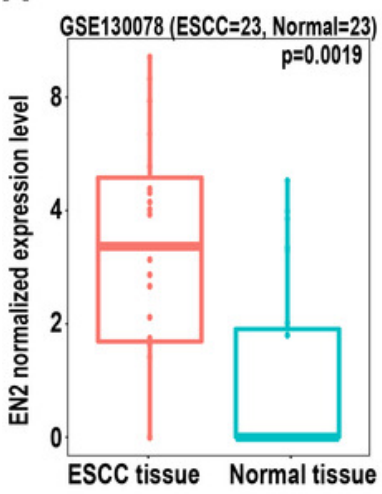

B

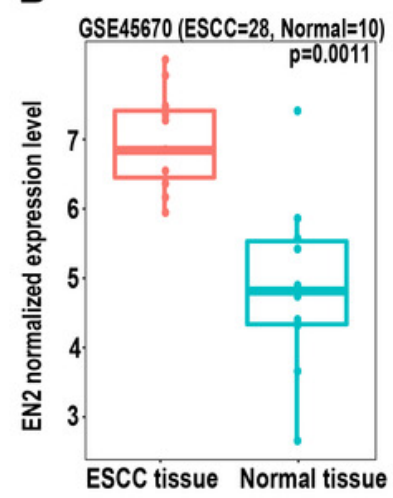

C

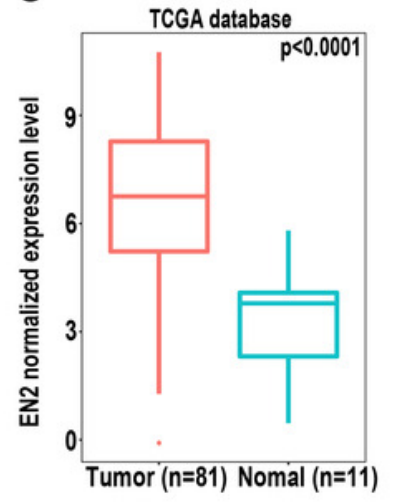

D

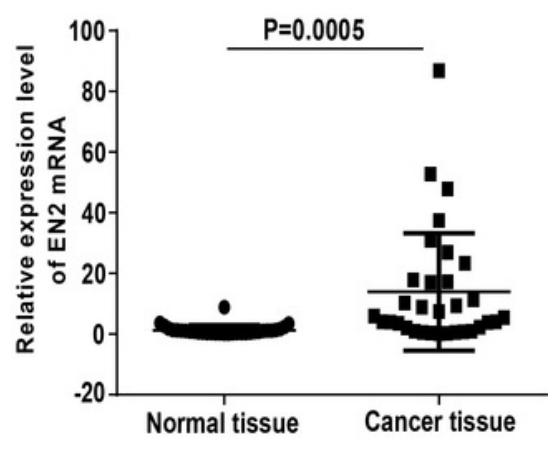

E

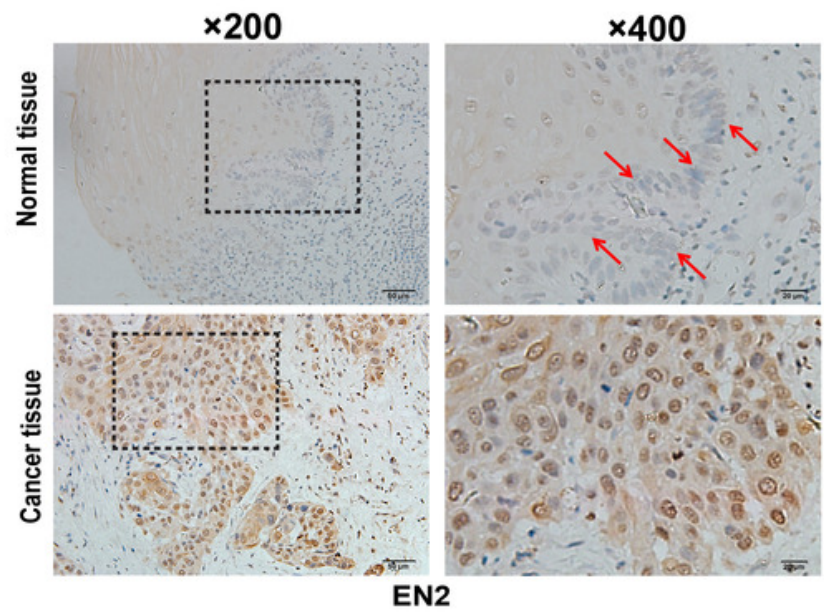

$\mathbf{F}$

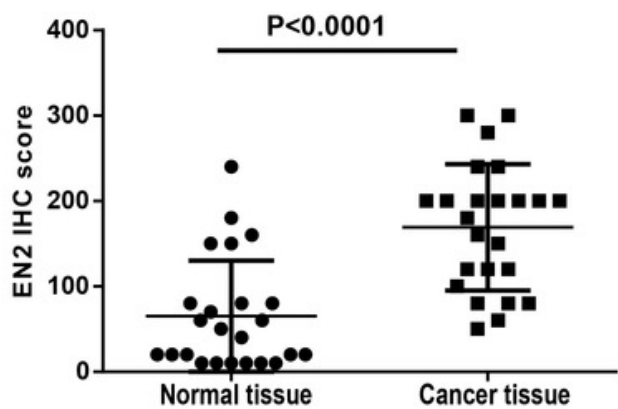




\section{Figure 2}

EN2 expression augments the growth and clonogenic abilities of ESCC cell lines.

(A) The mRNA expression of EN2 in three ESCC cell lines, in a mixture of total RNA extracted from normal esophageal samples adjacent to ESCC tumors of 32 patients, and in the SHEE control cell line was evaluated by means of qRT-PCR. (B) Eca109 and Kyse150 cells were infected with either EN2-containing or NEO-containing empty lentivirus particles. The mRNA expression level of EN2 was evaluated using qRT-PCR. (C, D) Fluorescent images of cancer cells stained with anti-EN2 antibody. EN2 staining is shown in green using FITC-labeled secondary antibody. Cell nuclei were stained blue (DAPI) and images were taken by confocal microscopy at $\times 20$ magnification. (E) EN2 promoted cell growth as demonstrated by CCK-8 assay. (F) EN2 promoted cell growth as determined by the IncuCyteZOOM $®$ live cell imaging assay. (G, H) Exogenous EN2 expression augmented the colony-forming ability of Kyse150 cell lines. (I, J) Exogenous EN2 expression augmented the colony-forming ability of Eca109 cell lines. $(* p<0.05, * * p<0.01, * * * p<0.001)$. 


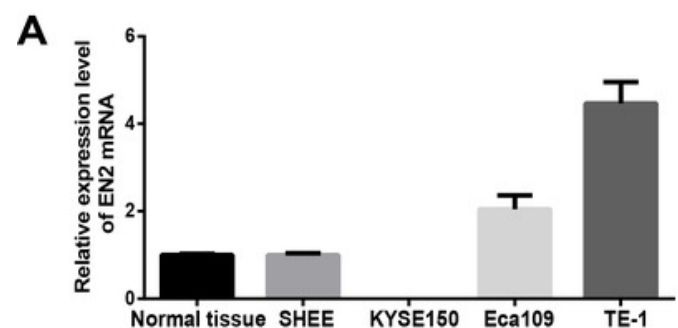

C
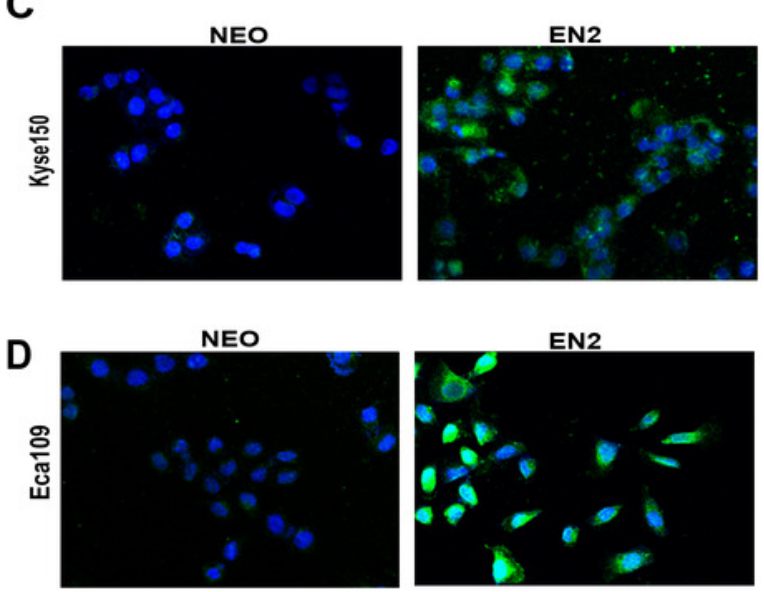

$\mathbf{F}$

Kyse150

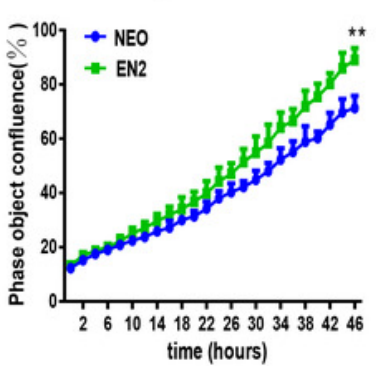

EN2

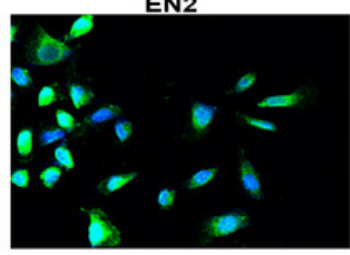

Eca109

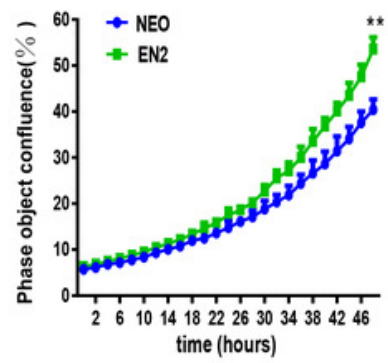

B

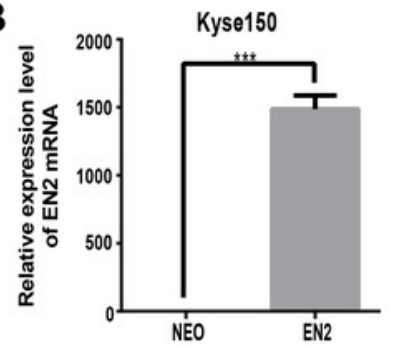

E

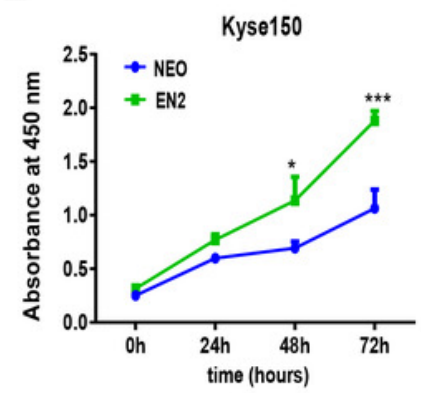

G
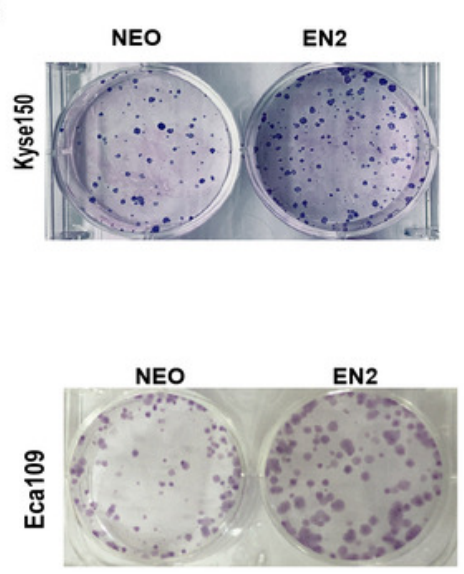
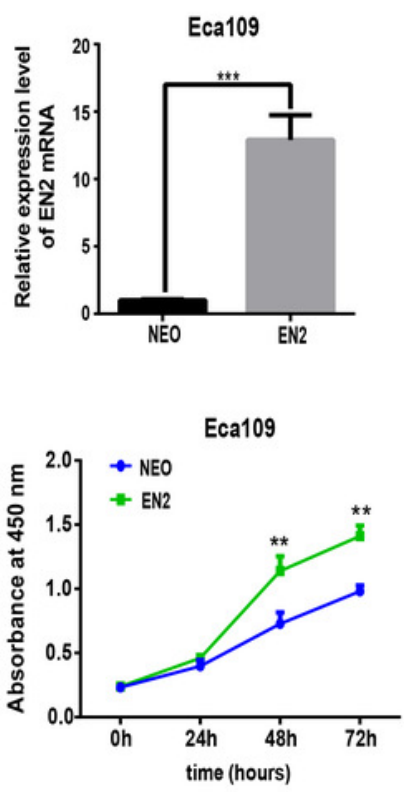

H

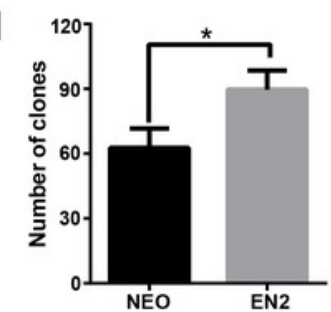

J

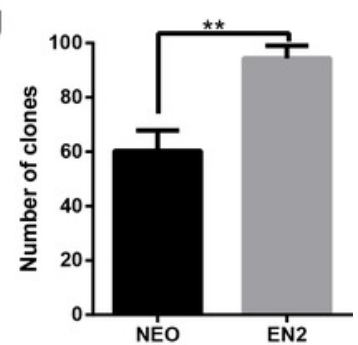




\section{Figure 3}

EN2 promotes the migration and invasion of ESCC cells.

(A, B) Migratory variation of Kyse150 cells expressing EN2 or NEO was evaluated using wound-healing assay. (C, D) Migratory variation of Eca109 cells expressing EN2 or NEO was evaluated using wound-healing assay. (E, F) Invasive variation of Kyse150 cells expressing EN2 or NEO was evaluated using Matrigel invasion assay. (G, H) Invasive variation of Eca109 cells expressing EN2 or NEO was evaluated using Matrigel invasion assay. Representative photographs (left) and quantification (right) are shown. The scale bars represent $300 \mu \mathrm{m}$. (** $p<0.01$, $* * * 0.001)$.

A

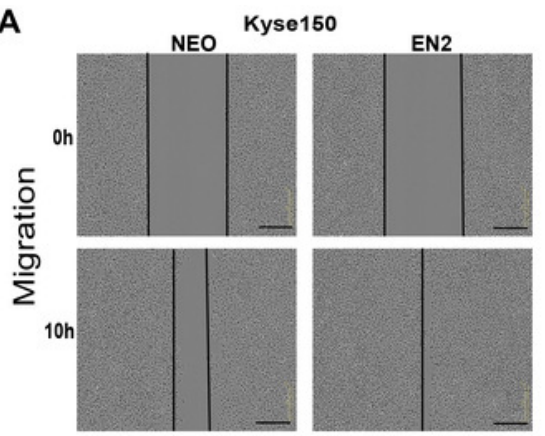

E

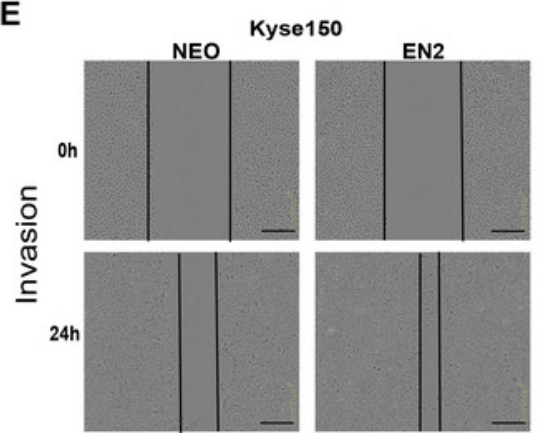

B

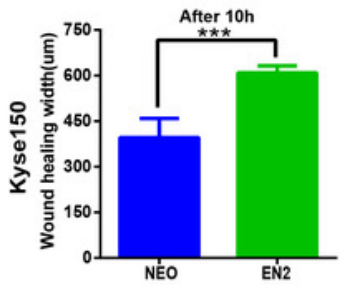

$\mathbf{F}$

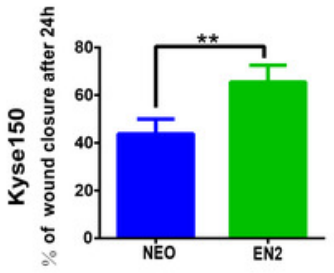

C

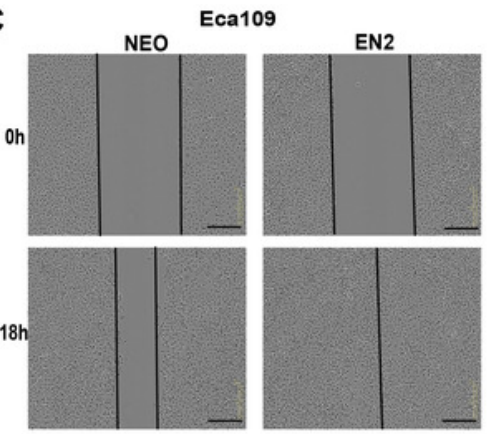

G

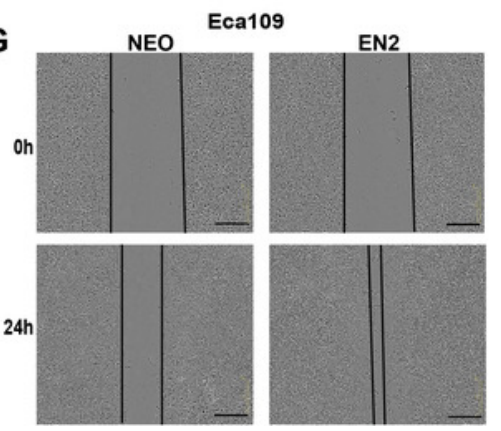

D

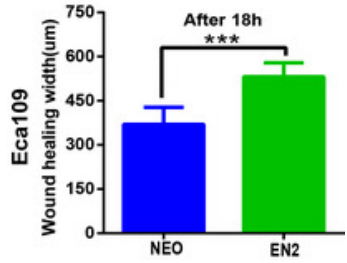

H

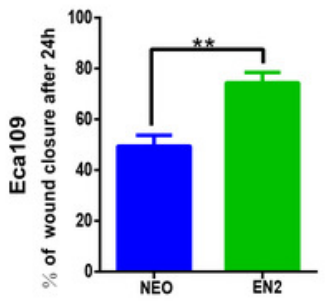




\section{Figure 4}

Silencing of EN2 negatively affects the proliferation, clonogenicity, migration, and invasion of TE-1 cells.

(A) Schematic representation of the CRISPR-Cas9 target site (marked in red), which is located at the first exon of the EN2 locus. The protospacer adjacent motif (PAM) is underlined. (B) The confirmation of effective disruption of the EN2 gene using the T7 endonuclease assay. The size of the T7 endonuclease I-digested DNA fragments is indicated on the right; lane M, 1 , and 2 indicate the DNA marker (DL1000, Takara), the sample from the parental TE- 1 cells transfected PX458 vector (control), and the sample from the EN2-disrupted cells. (C) The confirmation of effective disruption of EN2 protein using immunofluorescent staining. EN2 staining is shown in green (FITC-labeled secondary antibody). Cell nuclei are stained in blue (DAPI), images were taken by confocal microscopy at $\times 20$ magnification. (D) Disruption of EN2 decreases cell growth as determined by the live-cell imaging assay. (E, F) Disruption of EN2 hampers the colony-forming ability of TE-1 cells. (G, H) Disruption of EN2 suppresses the migratory potential of TE-1 cells. (I, J) Disruption of EN2 suppresses the invasive capacity of TE-1 cells. The scale bars equal $300 \mu \mathrm{m} .(* p<0.05, * * * p<0.001)$. 
A

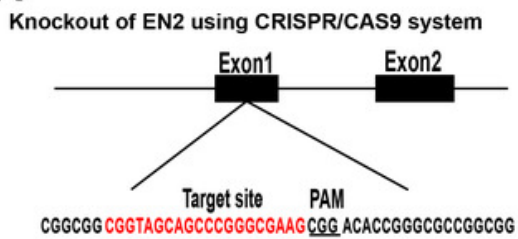

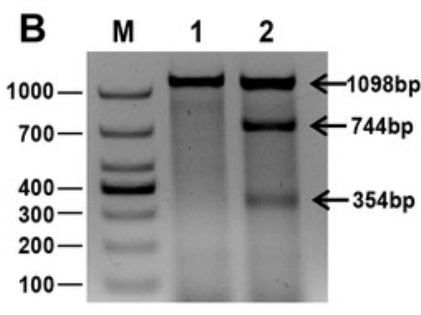
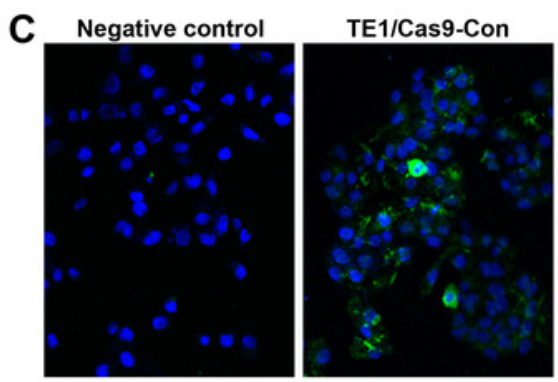

TE1/Cas9-EN2
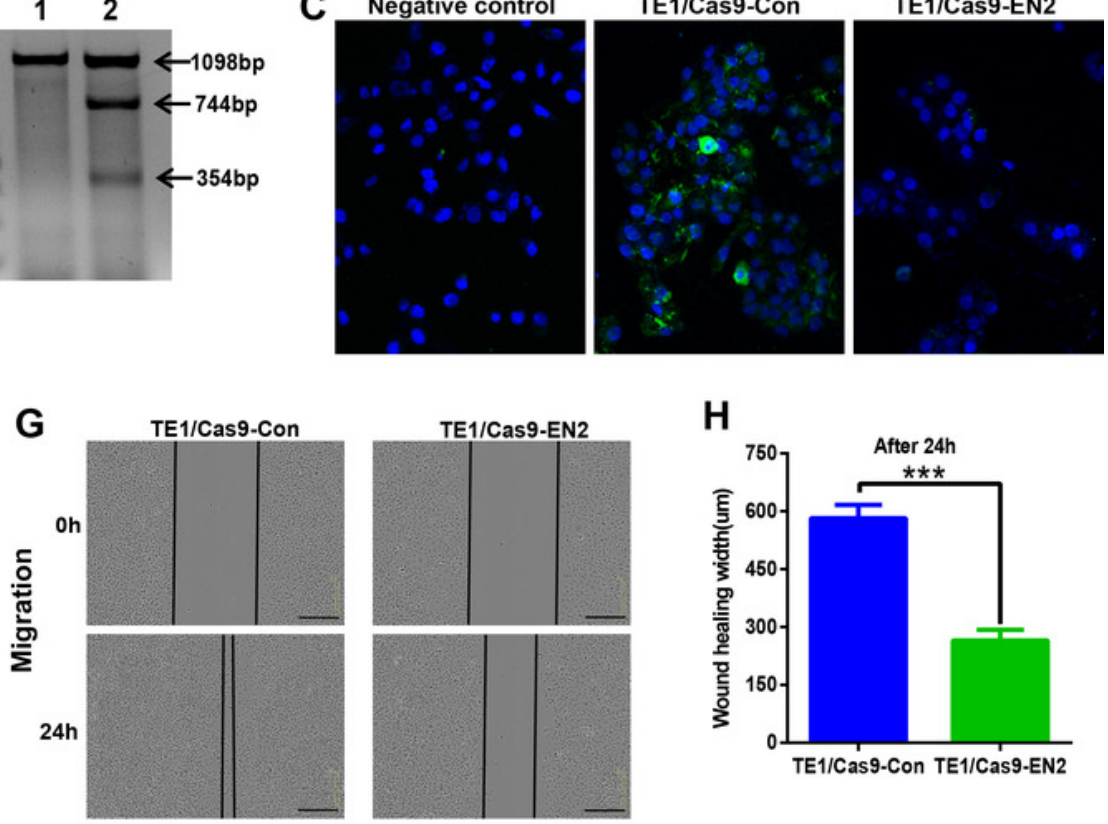

H
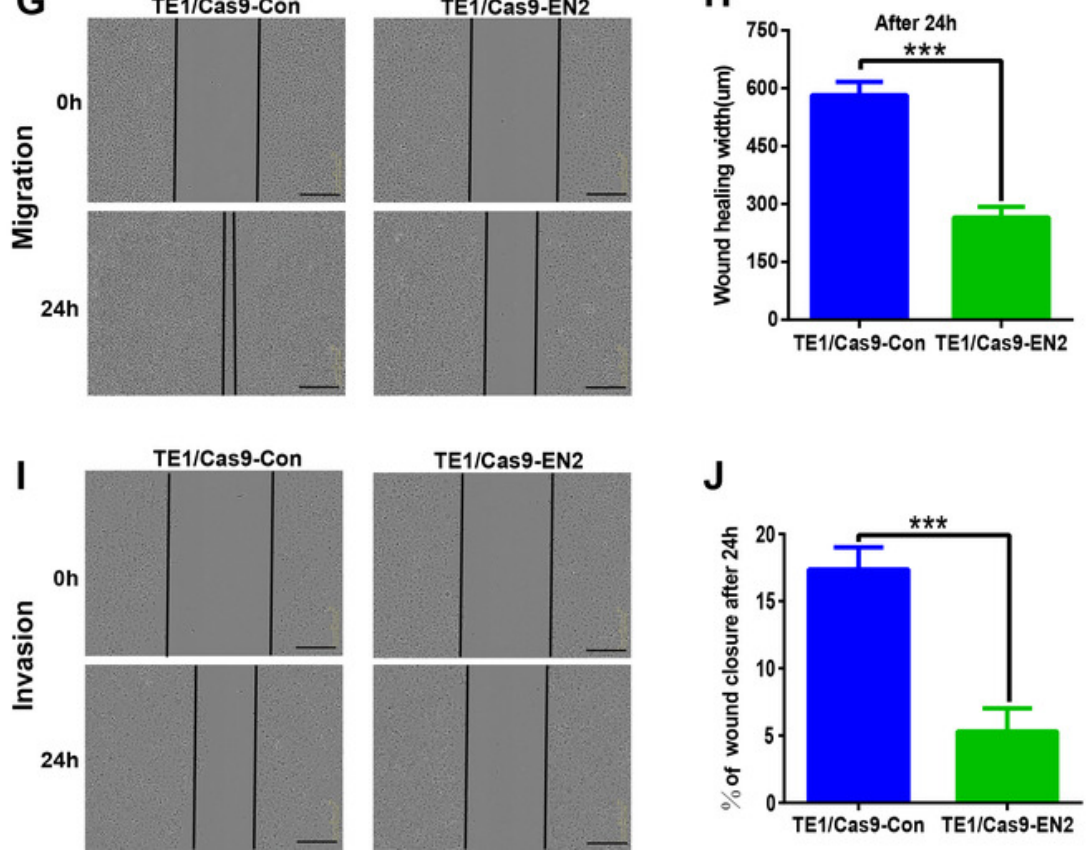
Figure 5

EN2 upregulates the expression of SPARC.

(A) Volcano maps show the differentially expressed genes in EN2-Eca109 as compared with the NEO-Eca109 cells. (B) Gene ontology (GO) analysis identified biological processes impacted by EN2 upregulated genes in Eca109 cells. (C) Gene ontology (GO) analysis identified biological processes impacted by EN2 downregulated genes in Eca109 cells. (D) Expression of the upregulated genes with high fold-change in EN2-Eca109 and NEO-Eca109 cells by means of qRT-PCR. (E) Examination of SPARC protein in EN2-Eca109 and NEO-Eca109 cells using the western blot assay. (F) Correlation analysis of EN2 and SPARC expression in ESCC tissue samples $(n=32)$.

A
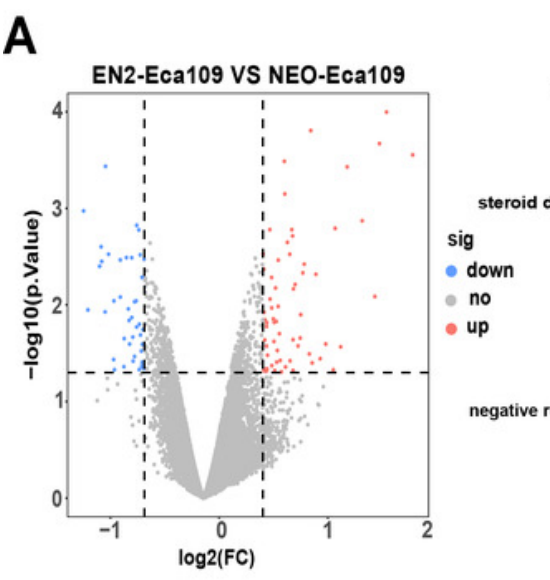

D

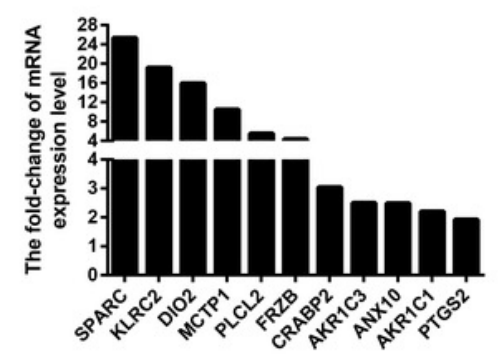

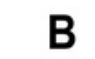

AMP-dependent protein kinase inhibitor activity protein kinase $A$ catalytic subunit binding extracellular matrix binding cAMP binding steroid dehydrogenase activity, NAD or NADP as acceptor steroid dehydrogenase activity protein serine/threonine kinase inhibitor activity cytokine receptor activity phosphoric diester hydrolase activity. cyclic nucleotide binding protein kinase inhibitor activity gative regulation of CAMP-dependent protein kinase activity kinase inhibitor activity complement receptor activity. protein kinase A binding inositol 1,4,6 trisphosphate binding GABA receptor binding growth factor binding. $3^{3}, 6^{6}$-cyclic-AMP phosphodiesterase activity

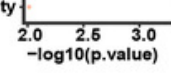

E

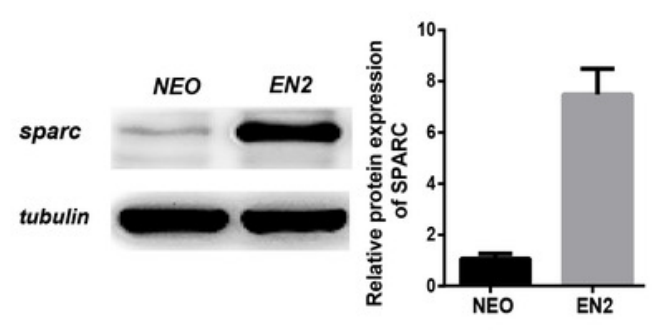
GO analysis
C EN2 downregulated genes in Eca109
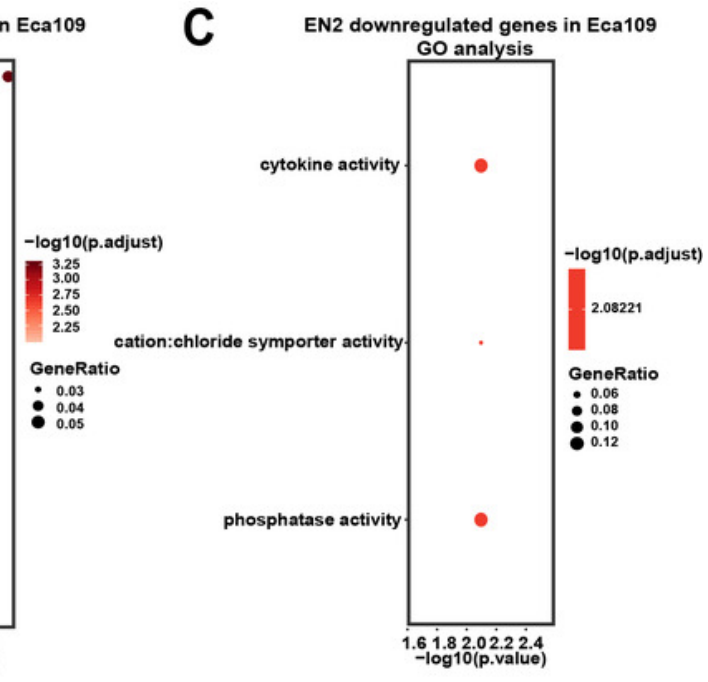

F

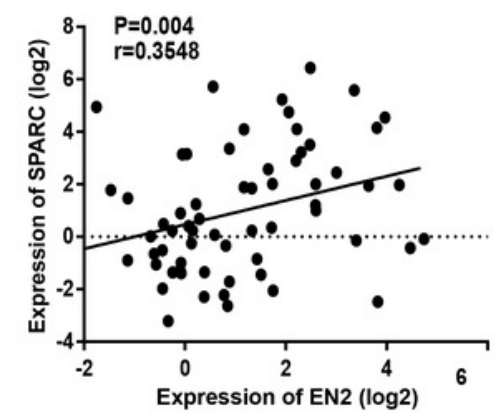




\section{Figure 6}

The homeodomain is essential for the SPARC induction and the protumor function of EN2.

(A) Schematic map of the two EN2 mutants with small deletions in the homeodomain region. $\alpha 1, \alpha 2$, and $\alpha 3$ indicate the three-helix structure in the homeodomain. (B) Confirmation of the expected mutations using the Sanger sequencing assay. The red triangles indicate the positions where part of the sequences are deleted. (C) The mRNA expressions of the EN2 and mutants were analyzed by qRT-PCR in Eca109 cells. EN2, Mut.1, and Mut.2 indicate the Eca109 cells infected with wild-type EN2, mutant 1, and mutant 2 expression lentivirus, respectively. (D) Analysis of the effect of the two mutants of EN2 on cell proliferation. (E, F) Analysis of the effect of the two mutants of EN2 on cell migration. (G, H) Analysis of the effect of the two mutants of EN2 on cell invasion. The scale bars represent $300 \mu \mathrm{m}$. (I, J) The mutations in the homeodomain attenuate the SPARC-inducing ability of the wild-type EN2 as determined by western blot assay. (* $p<0.05 ; * *<0.01)$. 
A

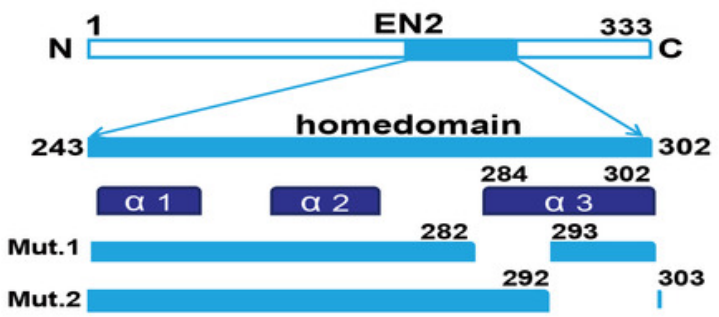

B

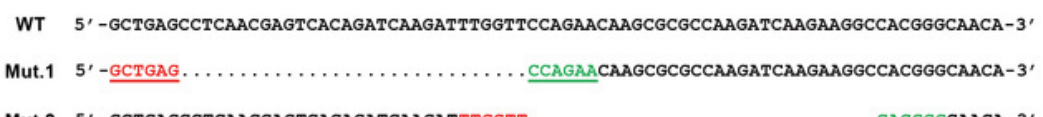

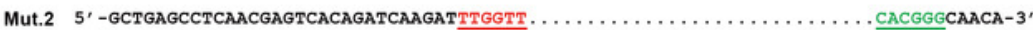

G Mut.1

GATCAAG Mut.2 2 TIGGTCACGGCACA

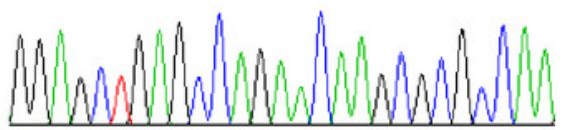

$\mathbf{E}$
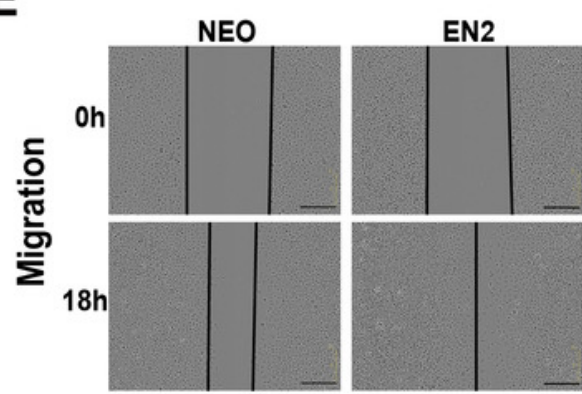

G
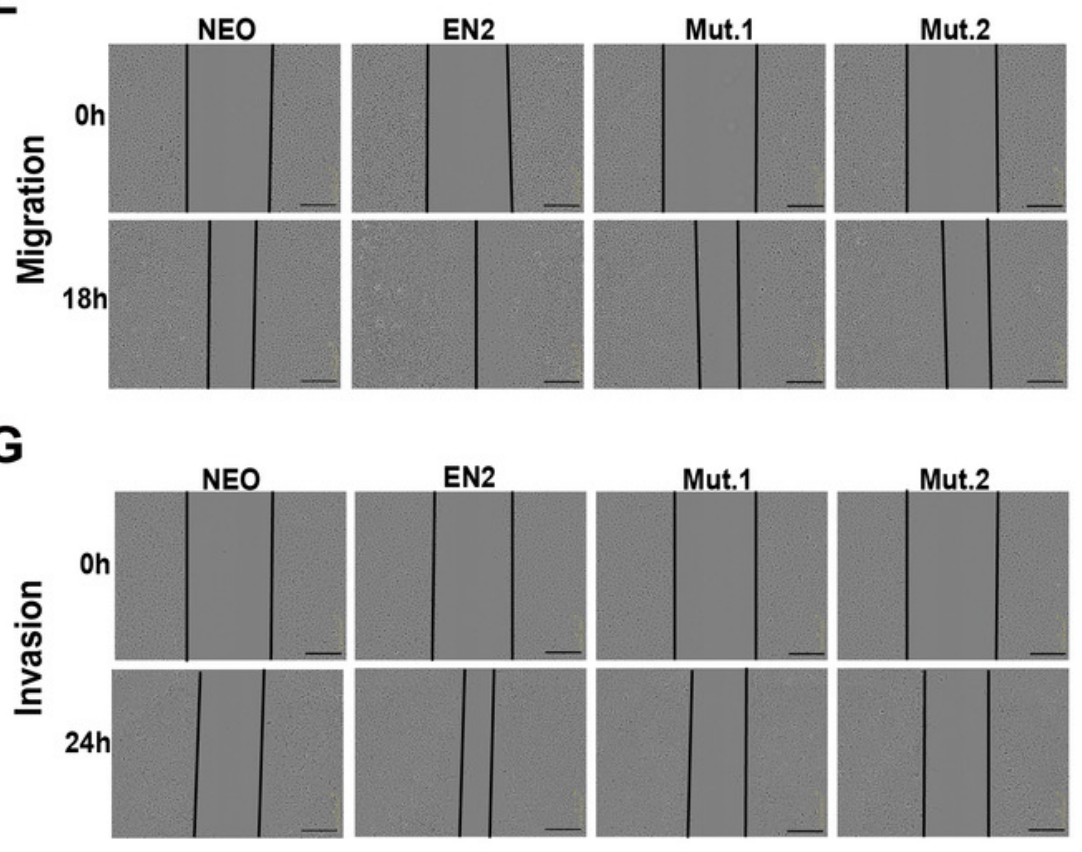

$\mathbf{F}$

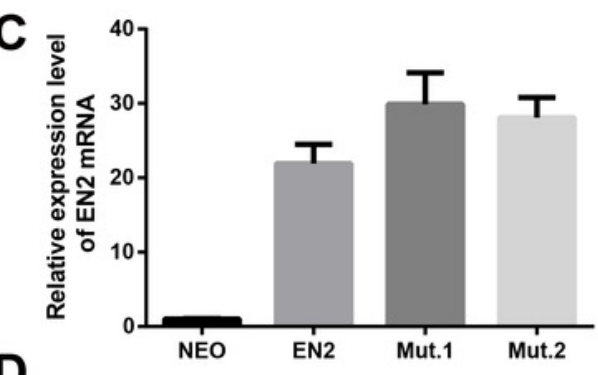

D
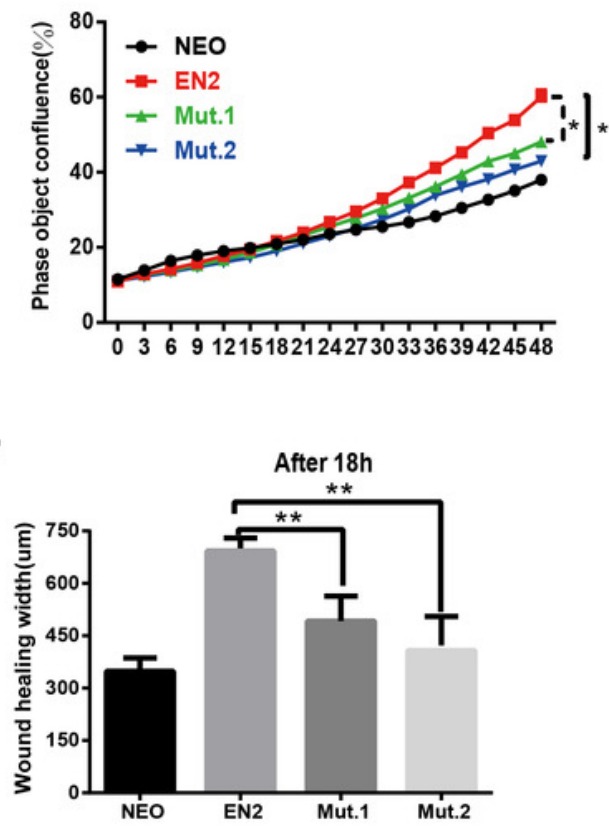

H
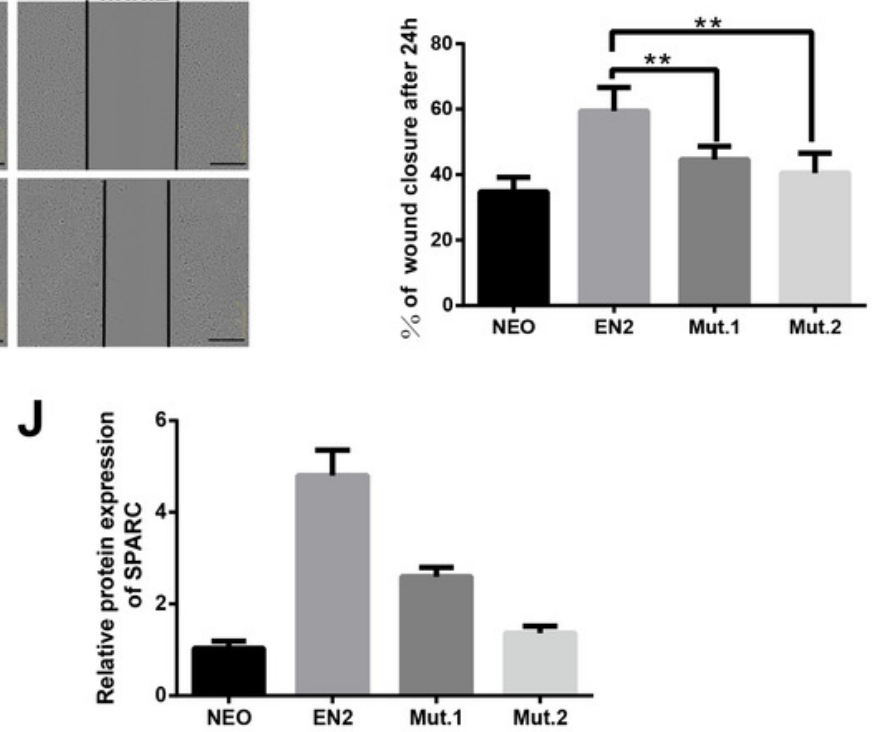


\section{Figure 7}

ShRNA-mediated knockdown of SPARC hampers the pro-oncogenic effect of EN2.

(A, B) Analysis of the efficiency of SPARC knockdown in EN2-Eca109 cells by means of the western blot assay. (C) The effect of SPARC knockdown on EN2-induced cell proliferation as determined by the live-cell imaging assay. (D, E) The effect of SPARC knockdown on EN2induced cell motility as determined by the wound healing assay. $(F, G)$ The effect of SPARC knockdown on EN2-induced cell invasion as determined by the Matrigel invasion assay. The scale bars represent $300 \mu \mathrm{m}$. (** $\mathrm{p}<0.01)$.

A

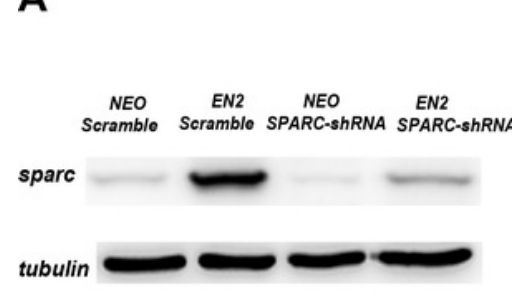

D
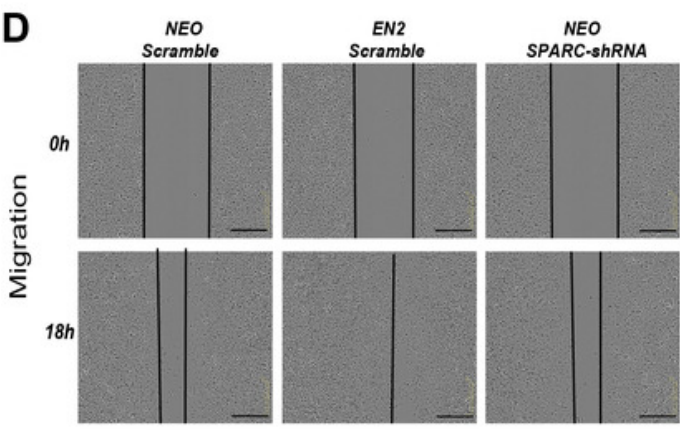

E

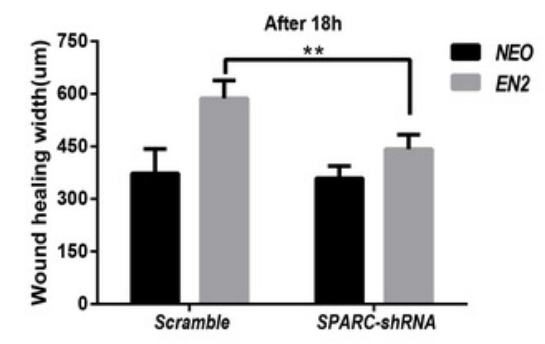

B

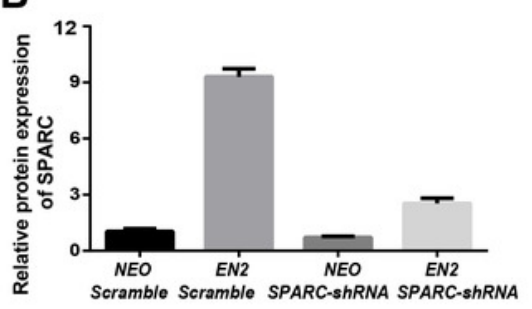

F

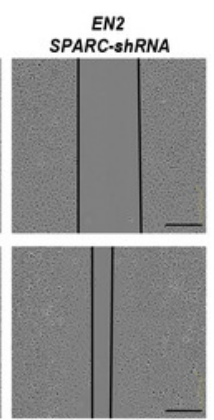

G
C

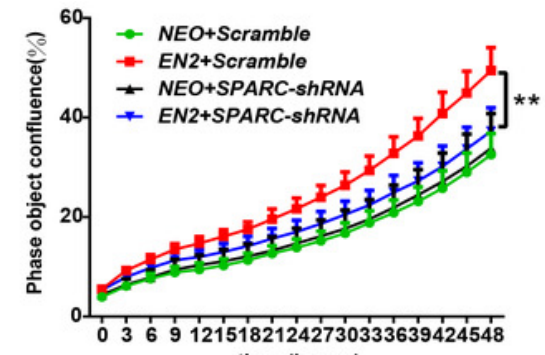

time (hours)
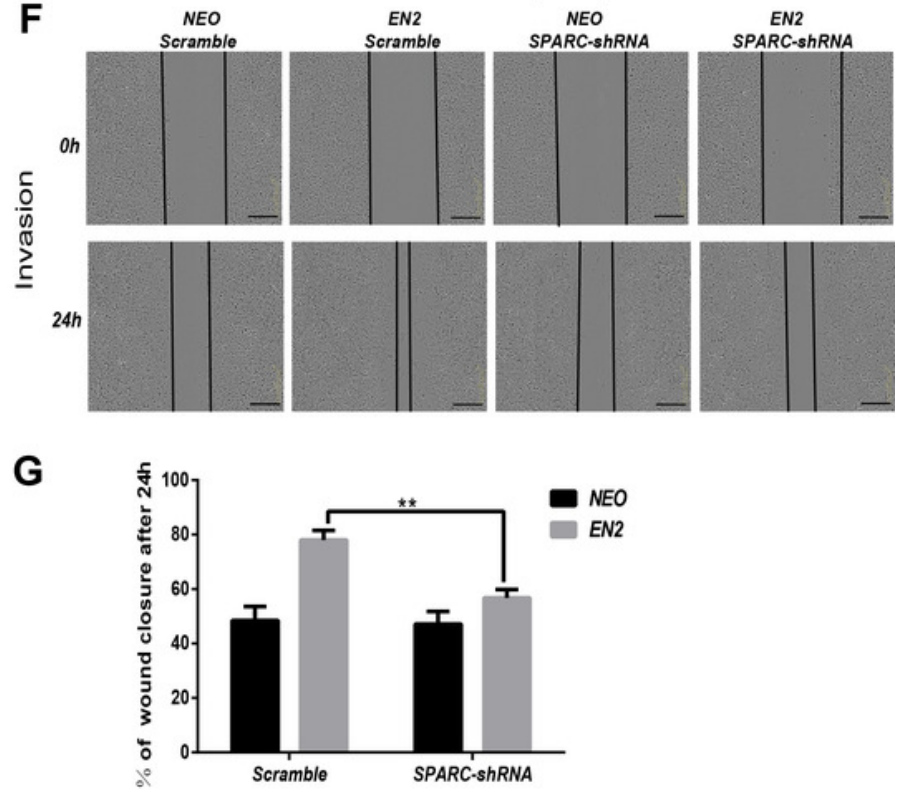